\title{
Pattern of subcarinal lymph node metastasis and dissection strategy for thoracic esophageal cancer
}

\author{
Qi-Xin Shang ${ }^{1 *}$, Yun-Cang Wang ${ }^{2 *}$, Yu-Shang Yang ${ }^{1}$, Wei-Peng Hu${ }^{1}$, Long-Qi Chen ${ }^{1}$ \\ ${ }^{1}$ Department of Thoracic Surgery, West China Hospital of Sichuan University, Chengdu, China; ${ }^{2}$ Department of Thoracic Surgery, Hospital of \\ Chengdu Office of People's Government of Tibetan Autonomous Region, Chengdu, China \\ Contributions: (I) Conception and design: LQ Chen; (II) Administrative support: LQ Chen; (III) Provision of study materials or patient enrollment: \\ YC Wang, QX Shang; (IV) Collection and assembly of data: QX Shang, YC Wang, WP Hu, YS Yang; (V) Data analysis and interpretation: YC \\ Wang, WP Hu, YS Yang; (VI) Manuscript writing: All authors; (VII) Final approval of manuscript: All authors. \\ \#These authors contributed equally to this work. \\ Correspondence to: Prof. Long-Qi Chen. Department of Thoracic Surgery, West China Hospital of Sichuan University. No. 37, Guoxue Alley, \\ Chengdu 610041, China. Email: drchenlq@scu.edu.cn.
}

Background: This study aimed to assess the role of subcarinal lymph nodes in lymph node metastasis in thoracic esophageal squamous cell carcinoma (ESCC) and to investigate the adequate range of lymph node dissection during esophagectomy.

Methods: This study included 782 thoracic ESCC patients who underwent esophagectomy between July 2008 and December 2010. The metastatic rate of subcarinal lymph nodes and their influencing factors were investigated. The outcome of subcarinal lymph node dissection was assessed using the efficacy index (the incidence of metastasis to a lymph node station (\%) multiplied by the 5-year survival rate (\%) of patients with metastasis to that lymph node station and divided by 100). Additionally, postoperative complications were compared between the subcarinal lymph node resection and reservation groups.

Results: The metastatic rates of subcarinal lymph nodes in the upper, middle, and lower thoracic ESCC were $8.3 \%$ (4/48), 19.1\% (79/414), and 16.2\% (23/142), respectively $\left(\chi^{2}=3.669, \mathrm{P}>0.05\right)$ and in T1, T2, T3, and T4 tumors were $0 \%(0 / 71), 4 \%(4 / 100), 22.2 \%(85 / 383)$, and $34 \%(17 / 50)$, respectively $\left(\chi^{2}=42.859\right.$, $\mathrm{P}<0.05)$. Tumor invasion and size were significantly correlated with metastasis. For upper thoracic ESCC with positive subcarinal lymph nodes, metastasis tendency was mainly to the lower mediastinum. In middle third esophageal cancer, after subcarinal lymph nodes were involved, metastasis to the lower mediastinal lymph nodes increased by nearly $50 \%$, and bidirectional metastasis increased by nearly three times compared with that before involvement. For lower third cancer with positive subcarinal lymph nodes, metastasis tendency was mainly to the upper mediastinum. The postoperative complication rates in the resection and reservation groups were as follows: overall, $19 \%$ and $14.6 \%$, respectively $(\mathrm{P}>0.05)$, and pulmonary, $10.3 \%$ and $7.3 \%$, respectively $(\mathrm{P}>0.05)$. The efficacy indexes of lymph node dissection at the upper, middle, and lower third esophagus were $0 \%, 7.6 \%$, and $27.5 \%$, respectively.

Conclusions: Dissection of subcarinal lymph nodes, which does not increase postoperative complications, should be performed routinely in lower thoracic ESCC after submucosal invasion of tumor; meanwhile, tumors larger than $3 \mathrm{~cm}$ should also result in subcarinal lymph node dissection in patients with a tumor located in the upper esophagus and T1-T2 ESCC.

Keywords: Esophageal carcinoma; metastasis; lymph node dissection; subcarinal lymph node; efficacy index; complication

Submitted Apr 27, 2020. Accepted for publication Aug 26, 2020.

doi: $10.21037 /$ jtd-20-1776

View this article at: http://dx.doi.org/10.21037/jtd-20-1776 


\section{Introduction}

Esophageal cancer is one of the most common malignancies worldwide, especially in some areas of Asia (1). In 2012, it was reported as the fourth lethal alimentary tract cancer in the United States (2). Currently, operation is the mainstay treatment, especially for resectable tumor patients (3). Presently, the 5-year survival rate has increased from $17.1 \%$ to $32 \%(3-5)$. However, $5-10 \%$ mortality and $50 \%$ morbidity after operation make the prognosis even more grim (6). Among various plausible causes, lymphadenectomy-induced complications and lymph node metastasis after operation should not be ignored. Many studies support the hypothesis that lymph node metastasis is responsible for postoperative relapse because the lymphatic route is one of the most important pathways for esophageal cancer metastasis (7). Theoretically, the more suspicious lymph nodes are removed, the more potential benefits such as more accurate staging, lower postoperative relapse, and higher survival rate may be achieved (8). However, postoperative complications and mortality might increase accordingly (9). Specifically, subcarinal lymph nodes are beneath the bronchial bifurcation near the pulmonary plexus of the vagus nerve. Hence, resection of these lymph nodes is associated with potential trauma to the trachea, such as thermal radiation injury possibly caused by electronic devices. In addition, the vagus nerve can be injured, resulting in sputum retention. This is detrimental for postoperative pulmonary function restoration. Additionally, the bronchial artery may be ligated during the lymphadenectomy procedure. Consequently, blood supply to the trachea and bronchus may be reduced partially. Practitioners are concerned about all these potential risks. Thus, there is dispute concerning whether extensive lymphadenectomy should be performed (10). An adequate range of lymph nodes dissection can maintain the balance between operative effects and postoperative complications/mortality. Therefore, exploring a normative lymphadenectomy based on reasonable consideration is much significant.

Unfortunately, several lymph nodes surround the esophagus and specific metastatic rule is obscure. Lymphatic vessels in the thoracic esophageal cancer are extensive, connecting the lymph nodes in the neck, mediastinum, and abdomen. Subcarinal lymph nodes, which are adjacent to bronchial bifurcation, might be a metastatic station. Hsu et al. reported that the metastatic rate of subcarinal lymph nodes was $23.8 \%$ (11). However, $\mathrm{Li}$ et al. found the rate to be $10.4 \%$ (12). Generally, subcarinal lymph nodes should be resected because they are regional lymph nodes of the esophagus (13), and their metastases are more likely to be associated with postoperative recurrence and metastasis (14). However, Li et al. suggested that the resection may be unnecessary in early stage and upper thoracic esophageal cancer (12). This inconclusiveness regarding lymphadenectomy of subcarinal lymph nodes has been a long-standing concern for practitioners.

Few studies have focused on these concerns and no standard dissection guideline is available for practitioners. Therefore, this study assessed the role of subcarinal lymph nodes in lymph node metastasis in thoracic esophageal squamous cell carcinoma (ESCC) and investigated the adequate range of lymph node dissection during esophagectomy. We present this article in accordance with the STROBE reporting checklist (available at http://dx.doi. org/10.21037/jtd-20-1776).

\section{Methods}

This study retrospectively evaluated patients with esophageal carcinoma who were admitted to our department and underwent esophagectomy. The study was conducted in accordance with the Declaration of Helsinki (as revised in 2013). The study was approved by the human participants committee of West China Hospital of Sichuan University (the ethical number: 2005-126). Preoperatively, permission for the use of patients resected specimens and written informed consents were obtained.

\section{Inclusion criteria}

We included patients with histologically confirmed thoracic ESCC who underwent radical esophagectomy as the primary therapy and had complete clinicopathological data.

\section{Exclusion criteria}

We excluded patients who had cervical esophageal cancer, had received neoadjuvant chemo/radiotherapy, or had ESCC combined with other malignancies.

\section{Patients}

A total of 931 patients with ESCC were admitted into our department from July 2008 to December 2010, of which only 782 (men: 656 and women: 126) were eligible 
for analysis. The median age of the patients was 59 years (range: 33-82 years). The clinicopathological stage of ESCC complied with the seventh edition American Joint Committee on Cancer tumor-node-metastasis staging criteria (2009) (15).

\section{Surgical procedure and pathology}

The surgical approach and lymphadenectomy's selection were depended on patients' preoperative examinations, at the same time, surgeons evaluated the patients' general condition and finally supplied for the patients the most appropriate surgical procedures. Generally, the McKeown esophagogastrectomy with three-filed lymph node dissection, the Sweet and Ivor-Lewis esophagogastrectomy with two-filed lymph node dissection may be performed. The lymph node identification and dissection for each patient were strictly followed by American Joint Committee on Cancer tumor-node-metastasis staging criteria (2009). Two experienced pathologists would fix the resected specimens, embedded and stained them with diaminobenzidine chromogen counterstained solution [1:50, EnVision TM Detection Kit, Gene Tech (Shanghai) Company Limited] and hematoxylin (Zhongshan Golden Bridge Biotechnology Co., Ltd, Beijing, China) subsequently. The routine way of assessing each specimen was adopted histologically, and the pathologists documented the extent and location of metastatic lymph nodes by examining the largest cross section of dissected lymph nodes.

\section{Spectrum of postoperative complications}

Postoperative pulmonary complication was defined as occurrence of any one of the following complications not because of anastomotic leak, massive pleural effusion, or nonpulmonary origin of infection (16): pneumonia, atelectasis/effusions, pleural effusion requiring placement of additional chest tubes, chylothorax, adult respiratory distress syndrome, respiratory failure requiring mechanical ventilation, and pulmonary embolism.

Anastomotic leakage was defined as the extravasation of water-soluble contrast medium and/or appearance of orally ingested methylene blue in the thoracic drainage (17).

Chylothorax was defined as a pleural effusion with presence of chylomicrons on lipoprotein electrophoresis or a triglyceride level higher than $110 \mathrm{mg} / \mathrm{dL}$ (18).
Recurrent laryngeal nerve paresis or palsy was diagnosed by the presence of hoarseness and aphonia (19).

Postoperative arrhythmias included sinus tachycardia, atrial fibrillation, multifocal atrial tachycardia, reentrant supraventricular tachycardia, atrial flutter, ventricular tachycardia, and heart blocks (20).

Wound infection, deep infection, wound hemorrhage, deep hemorrhage, chest infection, urinary infection, septicemia, wound dehiscence, deep venous thrombosis and pulmonary embolus, cardiac failure, impaired renal function, and hypotension were defined based on the Physiological and Operative Severity Score (21).

\section{Follow-up}

All patients were followed-up by telephone or interview at 3-month intervals for the first 2 postoperative years, at 6-month intervals for the following 3 years, and thereafter annually. Data regarding tumor status (tumor metastasis and recurrence), patient status (survival and death), and patients lost to follow-up were documented through outpatient follow-up as well as through telephone and letter followups. Survival time was measured from the date of operation to the date of death or last follow-up. The last general follow-up of survivors was conducted in the end of March 2016. The median follow-up time was 30.41 months (range: 1.06-62.86 months).

\section{Efficacy index}

The efficacy index $(22,23)$ is calculated as the incidence of metastasis to a lymph node station (\%) multiplied by the 5 -year survival rate (\%) of patients with metastasis to that lymph node station and divided by 100 .

\section{Statistical analysis}

Categorical data are expressed as numbers (percentage) and compared using the chi-square test or Fisher exact probability test. Continuous data are expressed as means \pm standard deviation and analyzed using grouped $t$-test. We performed the Kendall rank correlation test to determine intergroup differences in the rank data. A difference was considered statistically significant if the $\mathrm{P}$ value was $<0.05$. Data analysis was performed using Statistical Package for Social Sciences (SPSS) version 13 (SPSS, Chicago, IL, USA). 
Table 1 Comparison of clinicopathological demographics of patients between the resection and reservation groups

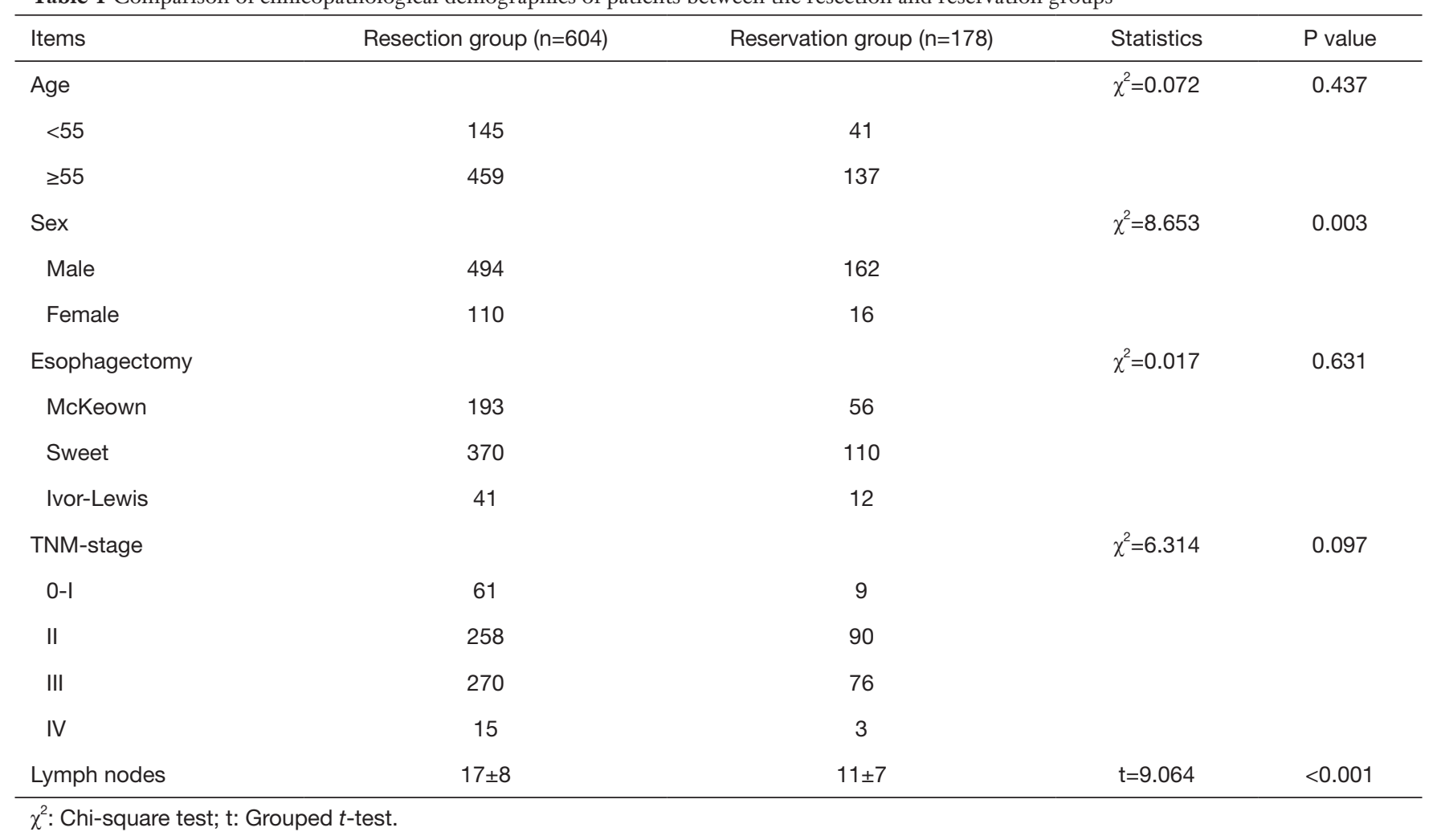

\section{Results}

\section{Patient characteristics and the effect of subcarinal lymph node metastasis}

Among 782 esophagectomy patients, 604 (77.2\%) patients underwent resection of subcarinal lymph nodes (resection group). The total number of subcarinal lymph nodes removed was 2,566 , with an average of five nodes per patient (range: $1-22)$. In the remaining $178(22.8 \%)$ patients, subcarinal lymph nodes were reserved (reservation group; Table 1).

Subcarinal lymph node metastasis was correlated with the length of tumor $(\mathrm{P}<0.001)$ and the depth of cancer invasion $(\mathrm{P}<0.001)$, among which we found patients with tumor size larger than $3 \mathrm{~cm}$ or T3-T4 ESCC got higher subcarinal lymph node metastasis than those with tumor size less than $3 \mathrm{~cm}$ or T1-T2 ESCC. In addition, pathological differentiation was an important factor in subcarinal lymph node metastasis. Although tumor location was not statistically significantly associated with subcarinal lymph node metastasis, the incidence of metastasis in upper thoracic ESCC was lower than that in middle and lower thoracic ESCC (Table 2).

\section{The role of subcarinal lymph nodes in lymph node metastasis}

Among 604 patients who underwent resection of subcarinal lymph nodes, $106(17.5 \%)$ patients had metastatic subcarinal lymph nodes. Fifty-six of 106 patients with positive subcarinal lymph nodes underwent subcarinal lymph nodes dissection. Among the 498 patients with negative subcarinal lymph nodes, 276 patients underwent subcarinal lymph nodes dissection. To evaluate the role of subcarinal lymph nodes in lymph nodes metastases, we summarized the data of 332 patients with subcarinal lymph nodes dissection. Considering subcarinal lymph nodes as the point of reference, the metastatic directions of other regional lymph nodes were divided as follows: upper mediastinum, lower mediastinum, both, and none (no other metastasis except the subcarinal nodes; Table 3). Additionally, the metastasis rate of the left gastric artery (No. 17), paraesophageal (No. $8 \mathrm{M}$ and $8 \mathrm{~L}$ ), gastric cardia (No. 16), upper paratracheal (No. 2L and 2R), lower 
Table 2 Correlation between subcarinal lymph node metastasis and pathological characteristics

\begin{tabular}{|c|c|c|c|c|c|}
\hline Pathological characteristics & Number of patients & Metastatic subcarinal LN (\%) & Correlation coefficient $(r)$ & Statistics & $P$ value \\
\hline Position of tumor & & & -0.015 & $\chi^{2}=3.669$ & 0.160 \\
\hline Upper & 48 & $4(8.3)$ & & & \\
\hline Middle & 414 & $79(19.1)$ & & & \\
\hline Lower & 142 & $23(16.2)$ & & & \\
\hline Length of tumor $(\mathrm{cm})$ & & & -0.142 & $\chi^{2}=16.887$ & $<0.001$ \\
\hline$<3.0$ & 133 & $8(6.0)$ & & & \\
\hline $3.0-5.0$ & 276 & $53(19.2)$ & & & \\
\hline $5.1-7.0$ & 153 & $35(22.9)$ & & & \\
\hline$>7.0$ & 42 & $10(23.8)$ & & & \\
\hline Depth of invasion & & & -0.251 & $\chi^{2}=42.859$ & $<0.001$ \\
\hline $\mathrm{T} 1$ & 71 & $0(0)$ & & & \\
\hline T2 & 100 & $4(4.0)$ & & & \\
\hline T3 & 383 & $85(22.2)$ & & & \\
\hline $\mathrm{T} 4$ & 50 & $17(34.0)$ & & & \\
\hline Differentiation & & & -0.054 & $\chi^{2}=4.750$ & 0.093 \\
\hline Well & 76 & 7 (9.2) & & & \\
\hline Moderately & 397 & $78(19.6)$ & & & \\
\hline Poorly & 131 & $25(19.1)$ & & & \\
\hline
\end{tabular}

paratracheal (No. 4L and 4R) and pulmonary ligament lymph nodes (No. 9) were 31\% (134/432), 28.2\% (128/454), $24.9 \%(87 / 350), 8.6 \%(18 / 209), 3.9 \%(6 / 155)$, and $3.9 \%$ $(5 / 129)$, respectively.

\section{Postoperative complications between two groups}

A total of 141 (18\%) patients experienced postoperative complications. Pulmonary complication was the most frequently encountered complication, occurring in 75 patients. No significant difference was not in the postoperative complication rates between both the groups (Table 4).

\section{Effect of subcarinal lymph node metastasis on survival}

The median follow-up time for resection group and reservation group are 30.30 months (range, 1.0662.86 months) and 23.06 months (range, 1.53-61.80). The overall survival was $48.337 \pm 1.698$ months in the resection group and $38.113 \pm 2.849$ months in the reservation group
$(\mathrm{P}<0.001$, Figure 1). With regard to tumor location, all patients in the resection group had a significantly better prognosis than those in the reservation group (all $\mathrm{P}<0.001$, Figure 2). The significantly better prognosis observed in the resection group was among patients with negative subcarinal lymph nodes rather than among those with positive subcarinal lymph nodes $(52.305 \pm 1.821$ vs. $19.918 \pm$ 1.698 months, $\mathrm{P}<0.001$, Figure 3).

However, for subcarinal lymph nodes at the upper, middle, and lower thirds of the esophagus in all patients, the resection rates were $70 \%, 74.9 \%$, and $76.9 \%$, respectively; the mean numbers $( \pm \mathrm{SD})$ of nodes resected were $3.9( \pm 2.8)$, $3.6( \pm 2.9)$, and $3.8( \pm 2.5)$, respectively; the frequencies of metastasis were $8.3 \%, 19.1 \%$, and $16.2 \%$, respectively; and the efficacy indexes of lymph node dissection were $0 \%$, $7.6 \%$, and $27.5 \%$, respectively (Table 5 ).

\section{Discussion}

The metastatic rate of subcarinal lymph nodes in thoracic ESCC was found to be $17.5 \%$, which is lower than that 
Table 3 Relationship between the metastatic direction of other regional lymph nodes and subcarinal lymph nodes

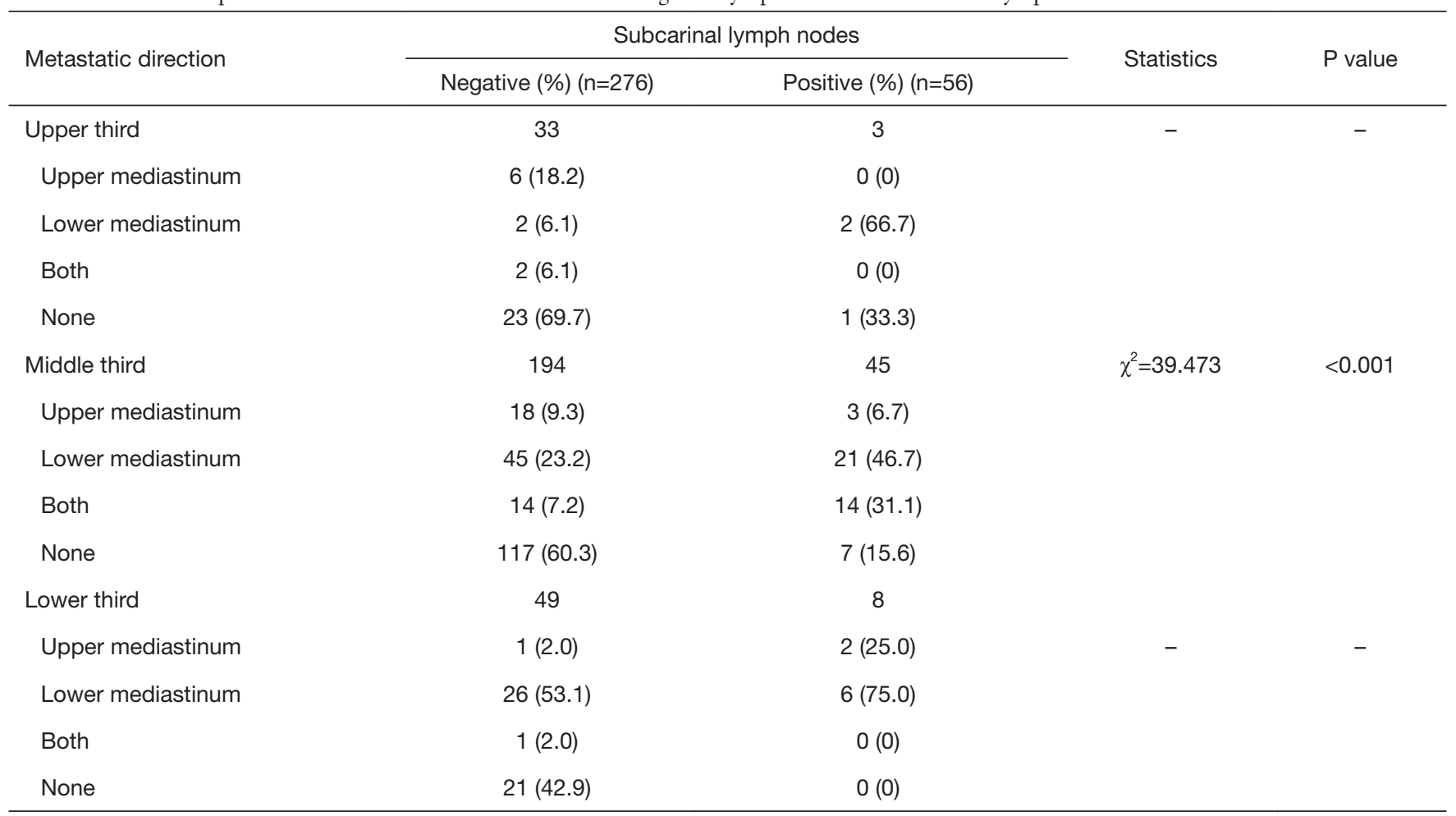

Table 4 Comparison of postoperative complications between the resection and reservation groups

\begin{tabular}{|c|c|c|c|c|}
\hline Complications & Resection group $(n=604)(\%)$ & Reservation group $(n=178)(\%)$ & Statistics & $P$ value \\
\hline Pulmonary infection & 43 & 7 & & \\
\hline Respiratory failure & 6 & 2 & & \\
\hline ARDS & 13 & 4 & & \\
\hline Chylothorax & $14(2.3)$ & $2(1.1)$ & $\chi^{2}=0.978$ & 0.323 \\
\hline Other complications & $22(3.6)$ & $3(1.7)$ & $\chi^{2}=1.701$ & 0.192 \\
\hline Overall & $115(19.0)$ & $26(14.6)$ & $\chi^{2}=1.828$ & 0.176 \\
\hline
\end{tabular}

reported by Hsu et al. (23.8\%) (11) and higher than that reported by Li et al. (10.4\%) (12). The plausible reason is the proportion of $\mathrm{T} 3$ and $\mathrm{T} 4$ patients in the study population. The proportion of $\mathrm{T} 3$ and $\mathrm{T} 4$ patients in Li et al. study was $61.9 \%(305 / 492)$, in Hus et al. study was $79.2 \%(80 / 101)$, and in our study was $71.7 \%$ (433/604). Moreover, Li et al. reported absence of positive subcarinal lymph nodes in upper esophageal cancer, which contrasted with the metastatic rate of $8.3 \%$ in our study. However, compared with metastasis of the paraesophageal lymph nodes among mediastinal lymph nodes, metastasis of the subcarinal lymph nodes in upper ESCC was less frequent. In addition, we demonstrated that both deeper tumor invasion and longer tumor length were correlated with high rate of subcarinal lymph node metastasis; however, histological differentiation did not 


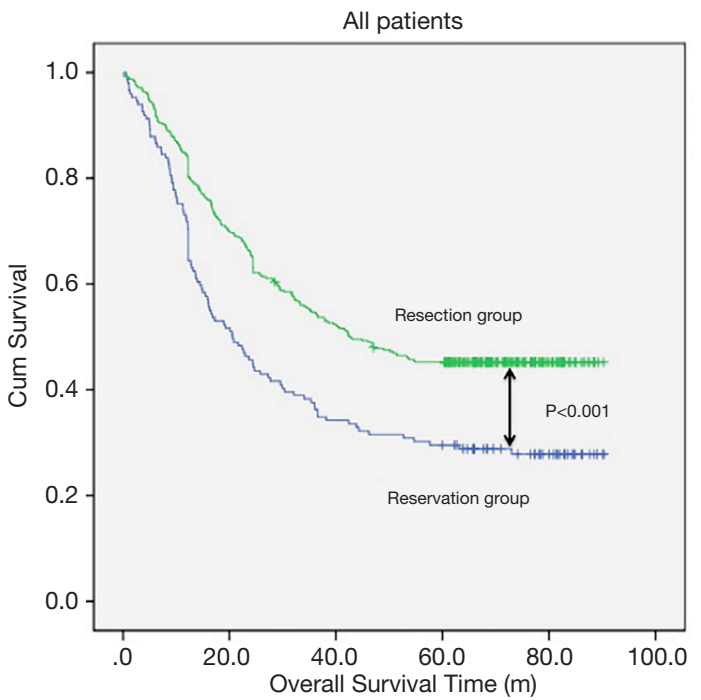

Figure 1 Survival analysis of Resection group \& Reservation group for all patients: $48.337 \pm 1.698$ months for dissection group vs. $38.113 \pm 2.849$ months for non-dissection group (Chi-square $=10.312, \mathrm{P}<0.001)$.

affect the metastatic rate. Although some studies have reported the same findings (12), a multivariate analysis by Zhu et al. denied the correlations (24). However, a further study with 1,812 consecutive patients confirmed the relationship (25). These discrepancies may have resulted from the complexity of subcarinal lymph nodes or limitation and differences in sample size. However, we found eight patients with tumor length $<3 \mathrm{~cm}$ and seven patients with histologically well-differentiated ESCC who had positive subcarinal lymph nodes and T3 or T4 stage. Therefore, tumor invasion rather than tumor length and differentiation might be a more prominent factor influencing the metastasis rate, which is consistent with the findings of Matsubara et al. (26). The possibility of lymph node metastases is low if the lesion is limited to the mucosa (27). However, the possibility of metastasis is highly elevated after submucosal invasion (28). Overall, subcarinal lymph nodes should be given prominence, especially in higher T-stage patients, regardless of the tumor location.

However, merely based on the metastatic rate, neither this nor other related studies could prove that subcarinal lymph nodes are sentinel lymph nodes (14). Because the distribution of lymph nodes in ESCC was unpredictable, we further investigated to clarify the role of subcarinal lymph nodes in lymph node metastasis, and believed it to be noteworthy.

We summarized the metastatic direction of lymph nodes surrounding subcarinal lymph nodes, considering subcarinal lymph node as the point of reference. In upper esophageal cancer, metastatic tendency was found to be downward after the subcarinal lymph nodes were involved, however, the number of patients with positive subcarinal lymph nodes was small, therefore, the tendency requires to be evaluated. In middle esophageal cancer patients with positive subcarinal lymph nodes, it was noted that both downward metastasis and increase in bidirectional metastatic tendency were highest. However, only upward metastasis decreased by nearly one third. Natsugoe $e t a l$. reported the reason for decrease in only upward metastasis as tumor invasion might be blocking the lymphatic pathway (29). The reason seems plausible and further study focused on this problem should be conducted. In lower ESCC patients, if the subcarinal lymph nodes were positive, both upward and downward metastasis increased and the former was found to be the main tendency, whereas the latter was found to be the main pattern. Moreover, if subcarinal lymph nodes were negative, other examined regional lymph nodes were rarely positive regardless of tumor location. Based on these obvious differences, it can be concluded that subcarinal lymph nodes are an important station in the lymphatic metastatic route. In addition, we found eight patients with solitary positive subcarinal lymph nodes. This can be explained as bidirectional or skip lymph node metastasis in esophageal cancer (30). The flow of lymph in the extramural lymphatic system from the distal esophagus was bidirectional, and the intramural lymphatic system communicated with different segments of the esophagus in the middle layer of muscularis mucosa. Moreover, the lymphatic pathway may be blocked by tumor invasion (29). Nevertheless, to some extent, it was still reasonable to consider subcarinal lymph node as a dependable indicator of other regional lymph node metastasis.

Lymphadenectomy may cause tissue injury and prolong operative time unavoidably. Postoperative complications resulting from the resection of subcarinal lymph nodes due to the special anatomic location should be considered. $\mathrm{Li}$ et al. reported that pulmonary complications in the resection group were considerably higher than those in the reservation group (12). On the contrary, in this study, no statistically significant differences were noted between the two groups for pulmonary complications 
A

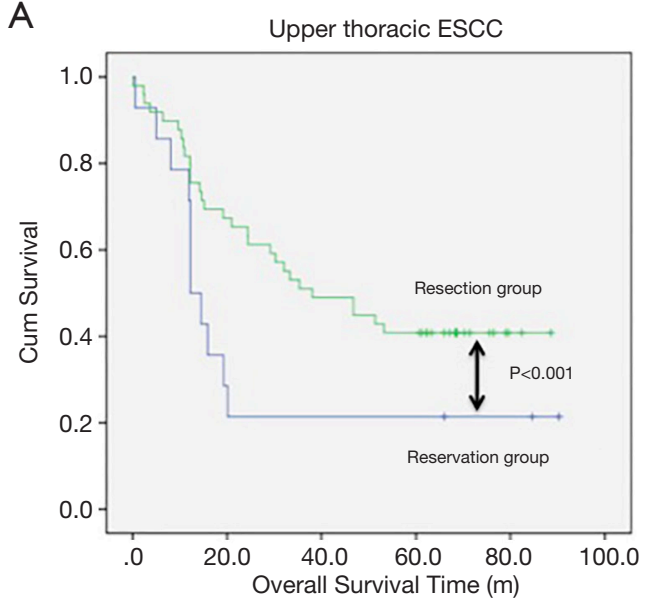

C

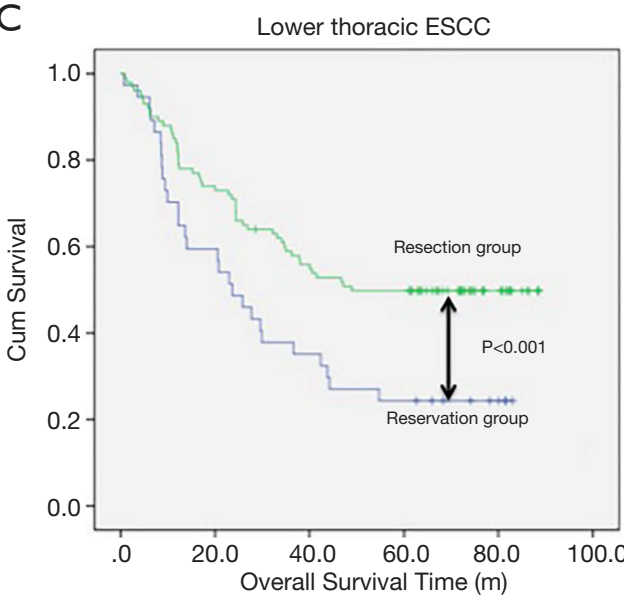

B

Middle thoracic ESCC

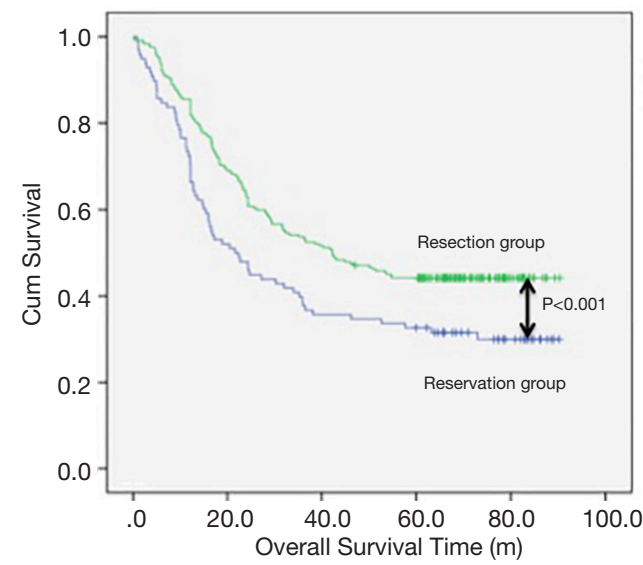

Figure 2 Survival analysis of Resection group \& reservation group for thoracic ESCC patients with regard to tumor location. (A) Patients with upper thoracic ESCC: $45.832 \pm 4.590$ months for dissection group vs. 28.743 \pm 8.691 months for non-dissection group (Chi-square $=10.1931, \mathrm{P}<0.001)$. (B) Patients with middle thoracic ESCC: 48.249 \pm 2.173 months for dissection group vs. $39.892 \pm 3.592$ months for nondissection group (Chi-square $=27.0464, \mathrm{P}<0.001$ ). (C) Patients with lower thoracic ESCC: $48.747 \pm 3.259$ months for dissection group vs. $35.085 \pm 4.897$ months for non-dissection group (Chi-square $=19.5780, \mathrm{P}<0.001)$.

as well as overall complications. This indicated that the special anatomic location of subcarinal lymph nodes and prolonged operative time might not be the main causes of postoperative complications. Actually, postoperative complications can be well prevented through meticulous maneuvers during operation and appropriate perioperative treatment strategies $(31,32)$. Many studies have reported no increase in complication rates after subcarinal lymphadenectomy (33).

In this study, the survival time was significantly better in the resection group than in the reservation group. In addition, the same trend was found when the patients were divided into three groups based on the tumor location. Similarly, the total number of lymph nodes removed was significantly higher in the resection group than in the reservation group. Therefore, we cannot judge the value of lymph node dissection. The low efficacy index $(0 \%)$ for subcarinal lymph nodes dissection in patients with upper thoracic ESCC indicates that subcarinal lymph node dissection does not contribute to the improved survival for these patients. The efficacy index in patients with middle thoracic ESCC was higher (7.6\%) and in those 
with lower thoracic ESCC was the highest (27.5\%). Based on our calculated efficacy index, subcarinal lymph node dissection can be omitted for patients with upper thoracic ESCC. However, Yukiko found that the characteristics of efficacy index were same in upper ESCC and lower ESCC patients (17).

Our study has some limitations. First, our study was a single-center retrospective study; therefore, analytical and selection biases were inevitable, however, in order to eliminate the interobserver variability of lymph nodes identification of our study, firstly, surgeons identified every station of lymph node and dissect them strictly followed by American Joint Committee on Cancer tumor-node-

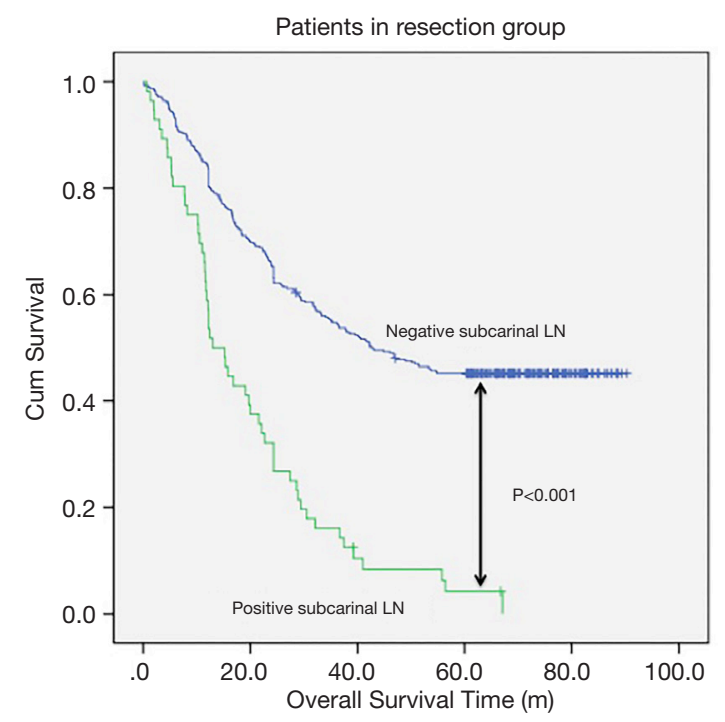

Figure 3 Survival analysis of Negative subcarinal LN \& Positive subcarinal LN for patients in Resection group: 52.305 1.821 months for patients with negative subcarinal LN vs. $19.918 \pm 1.698$ months for patients with positive subcarinal LN (Chi-square $=125.4935, \mathrm{P}<0.001$ ). metastasis staging criteria (2009), which guarantee the precise dissection of each station of lymph nodes. Secondly, the two experienced pathologists examined the resected lymph nodes histologically, which make sure the exact definition of subcarinal lymph nodes in our study. Second, the specific role of subcarinal lymph nodes in lymph node metastasis has not been investigated previously. In addition, the numbers of upper and lower ESCC patients were inadequate in this study. Thus, further investigation with a larger study population is warranted. Third, the prognostic significance of subcarinal lymph node metastasis was mainly focused on ESCC patients because esophageal cancer in most of the Chinese patients is squamous cell carcinoma; however, in most of the patients of the Western countries, esophageal cancer is adenocarcinoma. Thus, a large-scale multicenter, prospective study is warranted to verify the findings.

\section{Conclusions}

High incidence of subcarinal lymph node metastasis is observed in thoracic ESCC, especially among those with middle and lower esophageal cancer. The efficacy index of subcarinal lymph nodes dissection was higher for cancer of the lower esophagus than of the upper esophagus. The metastasis rate was obviously higher in T3-T4 stage patients than in T1-T2 stage patients. Therefore, we propose that in lower esophageal cancer, subcarinal lymph nodes dissection is necessary if the tumor has invaded the adventitia of the esophagus. However, whether the subcarinal lymph nodes dissection can be performed selectively in the upper esophageal cancer is still needed to be confirmed, but if the upper thoracic ESCC or T1T2 stage patients with tumor size larger than $3 \mathrm{~cm}$, the subcarinal lymph node dissection will be strongly recommended.

Table 5 Efficacy index of subcarinal lymph node stations in upper, middle, and lower thoracic ESCC patients

\begin{tabular}{|c|c|c|c|c|c|c|c|}
\hline \multirow[b]{2}{*}{ Tumor location } & \multicolumn{2}{|c|}{ AJCC 7th edition } & \multirow[b]{2}{*}{$\begin{array}{l}\text { Lymphadenectomy } \\
\text { rate }(\%)\end{array}$} & \multirow[b]{2}{*}{$\begin{array}{c}\text { Total number of lymph } \\
\text { nodes retrieved (mean } \pm \text { SD) }\end{array}$} & \multirow[b]{2}{*}{$\begin{array}{l}\text { Metastatic } \\
\text { rate }(\%)\end{array}$} & \multirow[b]{2}{*}{$\begin{array}{l}\text { 5-year DSS } \\
\text { rate }(\%)\end{array}$} & \multirow[b]{2}{*}{$\begin{array}{l}\text { Efficacy index } \\
(\%)\end{array}$} \\
\hline & Number & $\begin{array}{l}\text { Lymph node } \\
\text { station }\end{array}$ & & & & & \\
\hline Middle third & 7 & Subcarinal & 74.9 & $3.6 \pm 2.9$ & 19.1 & 0.4 & 7.6 \\
\hline Lower third & 7 & Subcarinal & 76.9 & $3.8 \pm 2.5$ & 16.2 & 1.7 & 27.5 \\
\hline
\end{tabular}

AJCC, American Joint Committee on Cancer; ESCC, esophageal squamous cell carcinoma; JES, Japan Esophageal Society; SD, standard deviation; DSS, disease-specific survival. 


\section{Acknowledgments}

Funding: None.

\section{Footnote}

Reporting Checklist: The authors have completed the STROBE reporting checklist. Available at http://dx.doi. org/10.21037/jtd-20-1776

Data Sharing Statement: Available at http://dx. doi. org/10. 21037/jtd-20-1776

Conflicts of Interest: All authors have completed the ICMJE uniform disclosure form (available at http://dx. doi. org/10. 21037/jtd-20-1776). The authors have no conflicts of interest to declare.

Ethical Statement: The authors are accountable for all aspects of the work in ensuring that questions related to the accuracy or integrity of any part of the work are appropriately investigated and resolved. The study was conducted in accordance with the Declaration of Helsinki (as revised in 2013). The study was approved by the human participants committee of West China Hospital of Sichuan University (the ethical number: 2005-126). Preoperatively, permission for the use of patients resected specimens and written informed consents were obtained.

Open Access Statement: This is an Open Access article distributed in accordance with the Creative Commons Attribution-NonCommercial-NoDerivs 4.0 International License (CC BY-NC-ND 4.0), which permits the noncommercial replication and distribution of the article with the strict proviso that no changes or edits are made and the original work is properly cited (including links to both the formal publication through the relevant DOI and the license). See: https://creativecommons.org/licenses/by-nc-nd/4.0/.

\section{References}

1 Yang BH, Parkin DM, Cai L, et al. Cancer burden and trends in the Asian Pacific Rim region. Asian Pac J Cancer Prev 2004;5:96-117.

2 Siegel R, Naishadham D, Jemal A. Cancer statistics, 2012. CA Cancer J Clin 2012;62:10-29.

3 Allum WH, Stenning SP, Bancewicz J, et al. Long-term results of a randomized trial of surgery with or without preoperative chemotherapy in esophageal cancer. J Clin Oncol 2009;27:5062-7.

4 Wei WQ, Yang J, Zhang SW, et al. Analysis of the esophageal cancer mortality in 2004 - 2005 and its trends during last 30 years in China. Zhonghua Yu Fang Yi Xue Za Zhi 2010;44:398-402.

5 Kelsen DP, Winter KA, Gunderson LL, et al. Longterm results of RTOG trial 8911 (USA Intergroup 113): a random assignment trial comparison of chemotherapy followed by surgery compared with surgery alone for esophageal cancer. J Clin Oncol 2007;25:3719-25.

6 Raymond D. Complications of esophagectomy. The Surgical clinics of North America 2012;92:1299-313.

7 Twine CP, Lewis WG, Morgan MA, et al. The assessment of prognosis of surgically resected oesophageal cancer is dependent on the number of lymph nodes examined pathologically. Histopathology 2009;5 5:46-52.

8 Tachibana M, Kinugasa S, Yoshimura H, et al. Clinical outcomes of extended esophagectomy with three-field lymph node dissection for esophageal squamous cell carcinoma. Am J Surg 2005;189:98-109.

9 Colvin H, Dunning J, Khan OA. Transthoracic versus transhiatal esophagectomy for distal esophageal cancer: which is superior? Interact Cardiovasc Thorac Surg 2011;12:265-9.

10 Collard JM. Lymph node excision in cancer of the esophagus. Ann Chir 1996;50:121-9.

11 Hsu CP, Hsu NY, Shai SE, et al. Pre-tracheal lymph node metastasis in squamous cell carcinoma of the thoracic esophagus. Eur J Surg Oncol 2005;31:749-54.

$12 \mathrm{Li} \mathrm{YF,} \mathrm{Hu} \mathrm{Y,} \mathrm{Lin} \mathrm{P,} \mathrm{et} \mathrm{al.} \mathrm{Significance} \mathrm{of} \mathrm{subcarinal} \mathrm{lymph}$ node selective dissection in thoracic esophageal carcinoma. Zhonghua yi xue za zhi 2010;90:2636-9.

13 Diseases JSfE. Guidelines for clinical and pathologic studies on carcinoma of the esophagus, ninth edition: part II. Esophagus 2004;1:107-25.

14 Ma H, Li Y, Ding Z, et al. The clinical significance of subcarinal lymph node dissection in the radical resection of oesophageal cancer. Interact Cardiovasc Thorac Surg 2013;16:839-43.

15 Edge SB, Compton CC. The American Joint Committee on Cancer: the 7th edition of the AJCC cancer staging manual and the future of TNM. Ann Surg Oncol 2010;17:1471-4.

16 Avendano CE, Flume PA, Silvestri GA, et al. Pulmonary complications after esophagectomy. Ann Thorac Surg 2002;73:922-6.

17 Xu QR, Wang KN, Wang WP, et al. Linear stapled 
esophagogastrostomy is more effective than handsewn or circular stapler in prevention of anastomotic stricture: a comparative clinical study. J Gastrointest Surg 2011;15:915-21.

18 Shah RD, Luketich JD, Schuchert MJ, et al. Postesophagectomy chylothorax: incidence, risk factors, and outcomes. Ann Thorac Surg 2012;93:897-903.

19 Pertl L, Zacherl J, Mancusi G, et al. High risk of unilateral recurrent laryngeal nerve paralysis after esophagectomy using cervical anastomosis. Eur Arch Otorhinolaryngol 2011;268:1605-10.

20 Slawski B, Pfeifer K. Postoperative Arrhythmias. In: Cohn SL. (editor.) Perioperative Medicine: Springer London, 2011: 431-9.

21 Copeland GP, Jones D, Walters M. POSSUM: a scoring system for surgical audit. Br J Surg 1991;78:355-60.

22 Sasako M, McCulloch P, Kinoshita T, Maruyama K. New method to evaluate the therapeutic value of lymph node dissection for gastric cancer. Br J Surg 1995;82:346-51.

23 Niwa Y, Koike M, Hattori M, et al. The Prognostic Relevance of Subcarinal Lymph Node Dissection in Esophageal Squamous Cell Carcinoma. Ann Surg Oncol 2016 23:611-8.

24 Zhu ZJ, Zhao YF, Hu Y, et al. Analysis of lymph node metastasis in the thoracic esophageal squamous cell carcinoma. Zhonghua Zhong Liu Za Zhi 2008;30:138-40.

25 Liu J, Hu Y, Xie X, et al. Subcarinal node metastasis in thoracic esophageal squamous cell carcinoma. Ann Thorac Surg 2012;93:423-7.

26 Matsubara T, Ueda M, Abe T, et al. Unique distribution

Cite this article as: Shang QX, Wang YC, Yang YS, Hu WP, Chen LQ. Pattern of subcarinal lymph node metastasis and dissection strategy for thoracic esophageal cancer. J Thorac Dis 2020;12(10):5667-5677. doi: 10.21037/jtd-20-1776 patterns of metastatic lymph nodes in patients with superficial carcinoma of the thoracic oesophagus. Br J Surg 1999;86:669-73.

27 Cense HA, van Eijck CH, Tilanus HW. New insights in the lymphatic spread of oesophageal cancer and its implications for the extent of surgical resection. Best Pract Res Clin Gastroenterol 2006;20:893-906.

28 Gotohda N, Nishimura M, Yoshida J, et al. The pattern of lymphatic metastases in superficial squamous cell carcinoma of the esophagus. Hepatogastroenterology 2005;52:105-7.

29 Natsugoe S. Experimental and clinical study on the lymphatic pathway draining from the distal esophagus and gastric cardia. Nihon Geka Gakkai Zasshi 1989;90:364-76.

30 Sato F, Shimada Y, Li Z, et al. Paratracheal lymph node metastasis is associated with cervical lymph node metastasis in patients with thoracic esophageal squamous cell carcinoma. Ann Surg Oncol 2002;9:65-70.

31 Farran Teixidor L, Llop Talaveron J, Galan Guzman M, et al. Surgical outcomes of esophageal cancer resection since the development of an oesophagogastric tumour board. Cir Esp 2013;91:517-23.

32 Shiozaki A, Fujiwara H, Murayama Y, et al. Perioperative outcomes of esophagectomy preceded by the laparoscopic transhiatal approach for esophageal cancer. Dis Esophagus 2014;27:470-8.

33 Shen Y, Zhang Y, Tan L, et al. Extensive mediastinal lymphadenectomy during minimally invasive esophagectomy: optimal results from a single center. J Gastrointest Surg 2012;16:715-21. 